\title{
The role of 3-dimensional ultrasound for the diagnosis of congenital uterine anomalies
}

\author{
Efraim Zohav ${ }^{1,2 *}$, Yaakov Melcer ${ }^{1,2}$, Ilan Tur-Kaspa ${ }^{3}$, Jacob Rabinson ${ }^{1,2}$, Eyal Y. Anteby ${ }^{1,2}$, Raoul Orvieto ${ }^{1,2}$ \\ ${ }^{1}$ Department of Obstetrics and Gynecology, Barzilai Medical Center, Ashkelon, Israel; \\ ${ }^{2}$ Ben-Gurion University of the Negev, Beer Sheva, Israel; \\ ${ }^{3}$ Institute for Human Reproduction (IHR), Department of Obstetrics and Gynecology, The University of Chicago, Chicago, USA. \\ Email: ${ }^{*}$ zohav@barzi.health.gov.il
}

Received 17 July 2011; revised 30 August 2011; accepted 18 September 2011.

\begin{abstract}
Objectives: To demonstrate the value of 3-dimensional (3-D) ultrasound (US) in the diagnosis of congenital uterine anomalies. Methods: Fifty one infertile patients referred to our US unit during 12 years period, with suspected diagnosis of congenital uterine anomalies by previous HSG or 2D US examinations, were evaluated by transvaginal 3-D US. The 3-D US diagnoses were compared to the initial HSG diagnosis, and to hysteroscopic evaluation when performed. Results: 3-D scan confirmed the initial HSG diagnosis in 27 out of $51(52.9 \%)$ women. The concordancy rates between the initial diagnosis by HSG and 3-D US results were $\mathbf{3 0 . 4 \%}$ for bicornuate uterus; $\mathbf{7 5 \%}$ for arcuate uterus; $83 \%$ for septate uterus; and $80 \%$ for unicornuate uterus. Of the 13 cases with normal HSG and suspicious 2-D US, $\mathbf{3 0 . 8} \%$ were found to be normal by 3D sonography. In cases where hysteroscopy was done, the results were $100 \%$ in concordance with the 3-D US evaluations. Conclusions: 3-D US is an accurate test for the assessment of uterine congenital anomalies. Its ability to concomitantly visualized, the external uterine contour with the uterine cavity on the same coronal plan, makes this noninvasive, easy to perform test the procedure of choice for the diagnosis of uterine anomalies.
\end{abstract}

Keywords: Mullerian Anomalies; 3-Dimensional (3-D) Ultrasonography; Hysterosalpingography; Hysteroscopy

\section{INTRODUCTION}

Congenital Mullerian duct anomaly is a common cause of infertility, first trimester abortion, preterm labor, and fetal malpresentations [1]. Its estimated prevalence varies between $0.1 \%-3 \%$ [2-4] in the general population and between $3 \%$ to $38 \%$ in patients with repeated spontaneous miscarriages or with infertility [5-10]. This dis- crepancy results from the heterogeneous population samples, the clinical diversity of Mullerian anomalies and the different diagnostic techniques used.

Several techniques are available for the evaluation of Mullerian duct anomaly, among which the traditional hysterosalpingography (HSG) is the most world-widely used, with its inherent limitation resulting from its inability to detect the external fundal contour of the uterus. Therefore, an invasive method which combines hysteroscopy and laparoscopy has been suggested as the gold standard for achieving a final diagnosis.

Recently, noninvasive tools such as three-dimensional (3-D) ultrasound and magnetic resonance imaging (MRI) have been added to our armamentarium, with their ability to demonstrate uterine contours, and therefore to improve the detection and differentiation between septate and bicornuate uteri.

The use of 3-D sonography with image reconstruction in the diagnosis of congenital uterine anomalies has already been described [11-14], enabling the assessment and accurate demonstration of both the internal and external contour of the uterine fundus which may easily be missed by the conventional 2-dimensional (2-D) US [15]. In the present study we aim to further evaluate the 3-D US imaging in the diagnosis of Mullerian duct anomaly, and its concordance with other imaging methods, such as 2-D US, HSG and hysteroscopy.

\section{PATIENTS AND METHODS}

The medical files of all women who underwent 3-D US during a 12 year period (1998-2010). For the purposes of this study, patients who were referred with the diagnosis of suspected Mullerian anomaly by previous HSG or 2-D US examinations were included in this study.

Patients underwent transvaginal 3-D US examinations using Voluson 530 and Voluson 730 expert (GE medical System) with transvaginal probes (3-D V-DW5-8B and RIC 5-9H respectively). All examinations were perfor- 
med by the same experienced sonographer (EZ) who was blinded to the clinical history and HSG/2D US evaluations.

Patients underwent 3-D US during the late follicular phase of the menstrual cycle [16], because of the increased thickness and echogenicity of the endometrium compared with the surrounding myometrium, which enables an easy and accurate interpretation.

Initial 3-D static volume acquisition with high resolution and sweep angle of 90 degree was done on 2-D ultrasound in mid-sagittal view of the uterus. All 3 perpendicular planes (sagittal, transverse and coronal sections) were displayed immediately after volume acquisition. The plans could be rotated simultaneously for the complete review of the anatomy. Reconstructed 3-D images in the coronal plan were further studied to differrentiate major uterine anomalies.

The diagnosis of Mullerian anomaly was made when good quality image showing both uterine cavity and fundus in coronal rendered plane was displayed. Mullerian anomalies were classified according to the American Fertility Society classification system [1]:

To distinguish bicornate from septate uteri we used criteria based on the presence or absence of fundal indentation of $1 \mathrm{~cm}$ or greater above the line connecting the two tips of the endometrial horns and divergent uterine horns with an intercornual distance exceeding $4 \mathrm{~cm}[17,18]$.

The criteria for the diagnosis of an arcuate uterus were a rounded endometrial indentation of the fundus and normal fundal contour.
Patients' 3-D US diagnoses were compared to their HSG and/or 2-D US imaging evaluations. Moreover, if a patient had undergone a further hysteroscopic evaluation, the result was also included in our analysis.

Data on patient age and infertility work-up evaluation were collected from their medical files. The results are expressed as means and standard deviations.

The study was approved by the institutional Clinical Research Committee.

\section{RESULTS}

Fifty one patients met the inclusion criteria. Mean patients' age at diagnosis was $32 \pm 6.9$ years. Mean patients' gravidity and parity were $0.88 \pm 1.38$ and $0.29 \pm 0.57$, respectively. Among the 51 women, 35 had a diagnosis of infertility with a mean duration of $4 \pm 2.7$ years and 16 suffered from repeated miscarriages.

The results of the initial 2-D US and HSG evaluations and the subsequent 3-D US and hysteroscopic examinations of the study group are shown in Figure 1.

3-D US confirmed the initial HSG diagnosis in 27 out of 51 (52.9\%) women. Among the 23 cases that were initially diagnosed by HSG as bicornuate uterus, the 3-D sonography confirmed the diagnosis in 7 (30.4\% concordance), while in the remaining 16 patients, 7 were diagnosed as having arcuate and 9 with septate uterus. Hysteroscopy confirmed the 3-D US diagnosis in 1 case of bicornuate uterus and 4 cases of septate uterus.

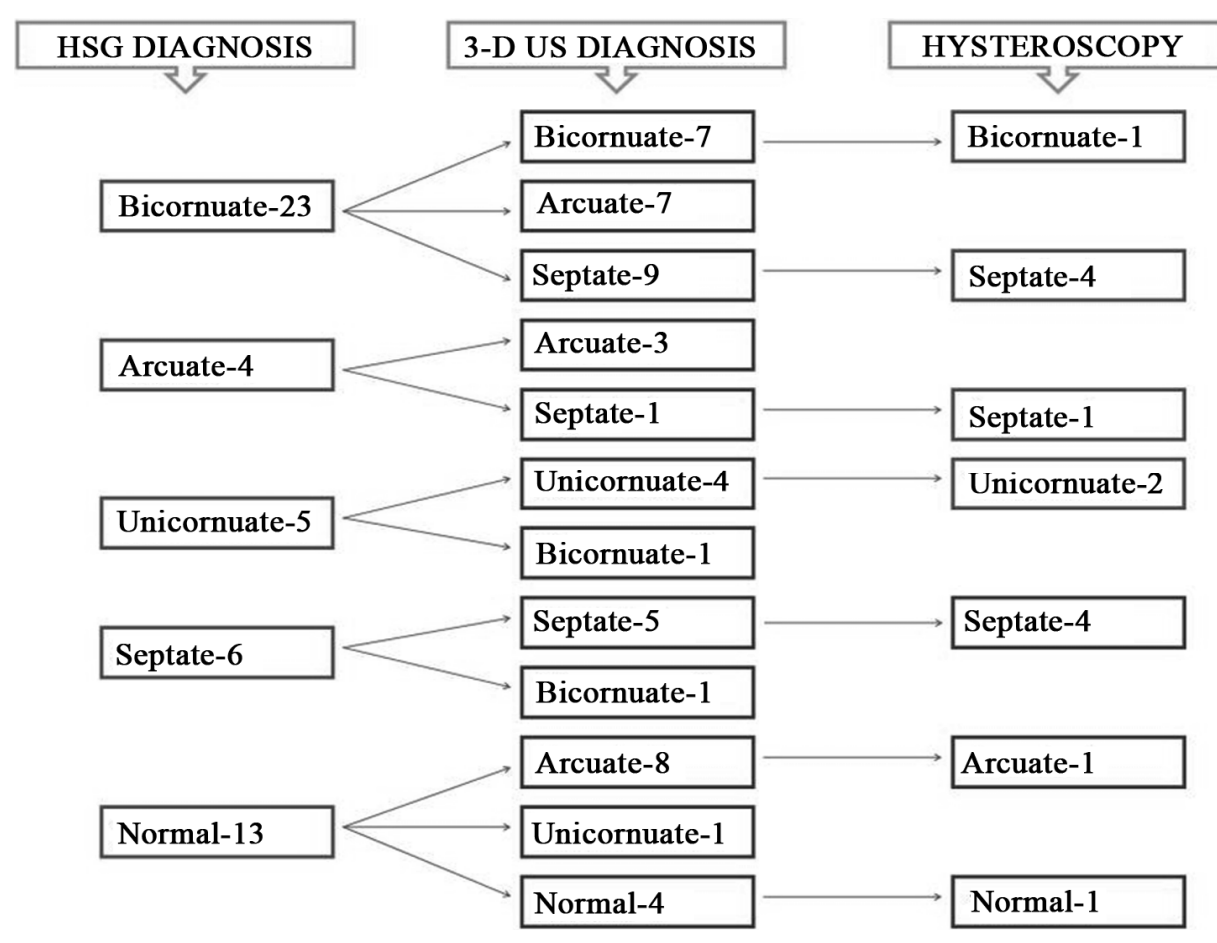

Figure 1. Two dimensional-US and HSG evaluations and the subsequent 3D-US and hysteroscopic examinations of the study group. 
Four patients were initially diagnosed by HSG as having an arcuate uterus. The 3-D sonography confirmed the diagnosis in 3 ( $75 \%$ concordance) out of 4 , and the fourth case was diagnosed as having septate uterus. Hysteroscopy confirmed the 3-D US diagnosis in the case of septate uterus. Moreover, in 6 cases that were initially diagnosed by HSG as suffering from septate uterus, the diagnosis was also confirmed by 3-D sonography in 5 cases (83\% concordance) and hysteroscopy (done in 4 cases).

Unicornuate uterus was initially diagnosed by HSG in 5 cases. While the 3-D sonography confirmed the diagnosis in 4 (80\% concordance), in the fifth case the diagnosis was a bicornuate uterus. Hysteroscopy confirmed the 3-D US diagnosis in 2 cases of unicornuate uterus.

Thirteen patients were referred to 3-D sonography because of suspicious Mullerian anomalies by 2-D sonography despite a normal HSG. 3-D US undertaken in these patients resulted in 4 normal cases (30.8\% concordance), 1 case of unicornuate uterus and 8 cases of arcuate uteri. Hysteroscopy confirmed the 3-D US diagnosis in 1 case of normal uterus and 1 case of arcuate uterus.

\section{DISCUSSION}

In the present study, 3-D US study of patients with suspected uterine mullerian anomalies by 2-D US and HSG confirmed the initial diagnosis in $52.9 \%$ of women. Clear visualization and satisfactory images of the uterine cavity were obtained in all patients without exclusion. The best concordance between 3-D US results and the initial 2-D US and HSG diagnosis was with regard to septate uterus (83\% concordancy), while in unicornuate, arcuate and bicornuate uteri the concordancy rates were $80 \%, 75 \%$ and $30.4 \%$, respectively. Of patients with normal HSG and suspicious 2-D US, 3-D US revealed uterine mullerian anomalies in $69.2 \%$. In the 14 patients undergoing hysteroscopic evaluation, all results were in agreement with the 3D US studies (100\% concordancy).

Our results support other studies demonstrating that 3-D US should become an important method for the assessment and the diagnosis of congenital uterine anomalies. In 1995 Jurkovic et al. [13] compared 2-D and 3-D ultrasound and HSG, finding both ultrasound modalities to have high predictive value for larger anomalies, especially in the differentiation of bicornuate and partially septate uteri. Raga et al. [14] found 3-D US to have a $91.6 \%$ accuracy in the study of the fundus and $100 \%$ in that of the cavity, compared to laparoscopy and HSG, respectively. Wu et al. [19], while comparing the 3-D US with laparoscopy and/or hysteroscopy, found 3-D ultrasound to have a $92 \%$ accuracy in the diagnosis of septate uterus and $100 \%$ for bicornuate uterus. Further studies comparing 3-D US to endoscopy revealed sensitivity of
$97 \%-100 \%$, specifity of $96 \%-100 \%$, positive predictive value of $92 \%$ and negative predictive value of $99 \%$ in the diagnosis of Mullerian anomalies [20,21] with a 96\% concordance between ultrasound and endoscopy with respect to the type of anomaly diagnosed [21].

Compared to 3-D US, the 2-D US, while being a sensitive method for detection of uterine anomalies $[15,18]$, it provides only a limited view of the uterine fundus and therefore cannot reliably differentiate between arcuate, bicornuate, and septate uteri [17,22]. HSG provides information on uterine cavity contour and tubal patency, however, its limitations results from its inability to classify the different subtypes of congenital uterine anomalies and to patients' exposure to ionizing radiation [23].

Hysteroscopy, on the other hand, is the gold standard diagnostic technique for endometrial cavity abnormalities, which can help revealing fundal septa suspected or unrecognized on HSG or ultrasound examinations [24].

3-D US overcomes the limitation of 2-D US and HSG by providing a coronal view of the uterus, which can rarely be seen by conventional 2-D US [13]. Recently [25], the value of 3D ultrasound in detecting congenital mullerian anomalies was found as accurate as MRI.

The coronal view enables the visualization of both the endometrial cavity and uterine fundus, thus providing all information necessary for a complete assessment of the nature and extent of the congenital anomaly and also facilitating the differential diagnosis between various fusion defects. The examination is performed in a standardized plane using corneal-interstitial portions of the fallopian tubes as the reference point, which enables quantitative description of uterine morphology. In addition, 3-D volumes can be stored on disk and re-examined later, which facilitates audit and independent verification of the diagnosis $[13,14,19]$.

In conclusion, 3-D US is an excellent, noninvasive, and accurate technology, which can serve as the gold standard in the assessment of congenital uterine anomalies.

\section{REFERENCES}

[1] Buttram, V.C. and Gibbons, W.E. (1979) Mullerian abnormalities: A proposed classification and analysis of 144 cases. Fertility and Sterility, 32, 40-46.

[2] Byrne, J., Nussbaum-Blask, A., Taylor, W.S., Rubin, A., Hill, M., O'Donnell, R. and Shulman, S. (2000) Prevalence of Mullerian duct anomalies detected at ultrasound. American Journal of Medical Genetics, 94, 9-12. doi:10.1002/1096-8628(20000904)94:1<9::AID-AJMG3 $>3.0 . \mathrm{CO} ; 2-\mathrm{H}$

[3] Ashton, D., Amin, H.K., Richart, R.M. and Neuwirth, R.S. (1988) The incidence of asymptomatic uterine anomalies in women undergoing transcervical tubal sterilization. Obstetrics Gynecology, 72, 28-30.

[4] Acien, P. (1997) Incidence of Mullerian defects in fertile 
and infertile women. Human Reproduction, 12, 13721376.

[5] Stampe Sorensen, S. (1988) Estimated prevalence of Mullerian duct anomalies. Acta Obstetricia et Gynecologica Scandinavica, 67, 441-445. doi:10.3109/00016348809004257

[6] Stray-Pedersen, B. and Stray-Pedersen, S. (1984) Etiologic factors and subsequent reproductive performance in 195 couples with a prior history of habitual abortion. American Journal of Obstetrics and Gynecology, 148, 140-146.

[7] Raga, F., Bauset, C., Remoh’I, J., Bonilla-Musoles, F., Simon, C. and Pellicer, A. (1997) Reproductive impact of congenital Mullerian anomalies. Human Reproduction, 12, 2277-2281. doi:10.1093/humrep/12.10.2277

[8] Makino, T., Hara, T., Oka, C., Toyoshima, K., Sugi, T., Iwasaki, K., Umeuchi, M. and Iizuka, R. (1992) Survey of 1120 Japanese women with a history of recurrent spontaneous abortions. European Journal of Obstetrics and Gynecology and Reproductive Biology, 44, 123-130. doi:10.1016/0028-2243(92)90057-6

[9] Clifford, K., Rai, R., Watson, H. and Reagan, L. (1994) An informative protocol for the investigation of recurrent miscarriage: Preliminary experience of 500 consecutive cases. Human Reproduction, 9, 1328-1332.

[10] Tur-Kaspa, I., Gal, M., Hartman, M., Hartman, J. and Hartman, A. (2006) A prospective evaluation of uterine abnormalities by saline infusion sonography (SIS) in 1009 women with infertility or abnormal uterine bleeding. Fertility and Sterility, 86, 1731-1735. doi:10.1016/j.fertnstert.2006.05.044

[11] Reute, K.L., Daly, D.C. and Cohen, S.M. (1989) Septate versus bicornuate uteri: Errors in imaging diagnosis. $R a-$ diology, 172, 749-752.

[12] Steiner, H., Staudach, A., Spitzer, D. and Schaffer, H. (1994) Three-dimensional ultrasound in obstetrics and gynecology: Technique, possibilities and limitations. $\mathrm{Hu}$ man Reproduction, 9, 1773-1778.

[13] Jurkovic, D., Giepel, A., Gruboeck, K., Jauniaux, E., Natucci, M. and Campbell, S. (1995) Three-dimensional ultrasound for the assessment of uterine anatomy and detection of congenital anomalies: A comparison with hysterosalphingography and two dimensional sonography. Ultrasound Obstetrics Gynecology, 5, 233-237. doi:10.1046/j.1469-0705.1995.05040233.x

[14] Raga, F., Bonilla-Musoles, F., Blanes, J. and Osborne, N.G. (1996) Congenital mullerian anomalies: Diagnostic accuracy of three-dimensional ultrasound. Fertility and Sterility, 65, 523-528.

[15] Nicolini, U., Bellotti, M., Bonazzi, B., Zamberletti, D. and Candiani, G.B. (1987) Can ultrasound be used to screen uterine malformations? Fertility and Sterility, $\mathbf{4 7}$ 89-93.

[16] Caliskan, E., Ozkan, S., Cakiroglu, Y., Sarisoy, H.T., Corakci, A. and Ozeren, S. (2010) Diagnostic accuracy of real-time 3D sonography in the diagnosis of congenital Mullerian anomalies in high-risk patients with respect to the phase of the menstrual cycle. Journal of Clinical Ultrasound, 38, 123-127.

[17] Pellerito, M.S., McCarthy, S.M., Doyle, M.B., Glickman, M.G. and DeCherney, A.H. (1992) Diagnosis of uterine anomalies: Relative accuracy of MR imaging, endovaginal sonography and hysterosalpingography. Radiology, 183, 795-800.

[18] Fedelee, L., Dorta, M., Brioschi, D., Massari, C. and Candiani, G.B. (1989) Magnetic resonance evaluation of double uteri. Obstetrics Gynecology, 74, 844-847.

[19] Wu, M.H., Hsu, C.C. and Huang, K.E. (1997) Detection of congenital mullerian duct anomalies using three-dimensional ultrasound. Journal of Clinical Ultrasound, 25, 487-492. doi:10.1002/(SICI)1097-0096(199711/12)25:9<487::AID -JCU4>3.0.CO;2-J

[20] Mohamed, M., Momtaz, M.D., Alaa, N., Ebrashy, M.D., Ayman, A. and Marzouk, M.D. (2007) Three-dimensional ultrasonography in the evaluation of the uterine cavity. MEFS Journal, 12, 41-46.

[21] Ghi, T., Casadio, P., Kuleva, M., Perrone, A.M., Savelli, L., Gianchi, S., Pelusi, C. and Pelusi, G. (2009) Accuracy of three-dimensional ultrasound in diagnosis and cla-ssification of congenital uterine anomalies. Fertility and Sterility, 92, 808-813. doi:10.1016/j.fertnstert.2008.05.086

[22] Alc'azar, J.L. (2005) Three-dimensional ultrasound in gynecology: Current status and future perspectives. $\mathrm{Cu}$ rrent Women's Health Review, 1, 1-14. doi:10.2174/1573404052950221

[23] Whitehouse, G.H. and Wright, C.H. (1992) Imaging in gynaecology. In: Grainger, R. G., and Allison, D. J., Eds., Diagnostic Radiology, 1825-1853.

[24] Sorensen, S.S. (1987) Hysteroscopic evaluation and endocrinological aspects of women with mullerian anomalies and oligomenorrhea. International Journal of $\mathrm{Fe}$ rtility, 32, 445-452.

[25] Bermejo, C., Martínez Ten, P., Cantarero, R., Diaz, D., Pérez Pedregosa, J., Barrón, E., Labrador, E. and Ruiz López, L. (2010) Three-dimensional ultrasound in the diagnosis of Müllerian duct anomalies and concordance with magnetic resonance imaging. Ultrasound Obstetrics Gynecology, 35, 593-601. doi:10.1002/uog.7551 